\title{
Embryonic Brain's Spontaneous Activity Promote Synesthesia?
}

\author{
J. Shashi Kiran Reddy ${ }^{1 *}$ and Georg Northoff $2,3,4,5$
}

\section{Affiliations:}

${ }^{1}$ Consciousness Studies Programme, National Institute of Advanced Studies (NIAS), Bengaluru, India.

${ }^{2}$ Mental Health Center, Zhejiang University School of Medicine, Hangzhou, Zhejiang, China;

${ }^{3}$ Mind, Brain Imaging and Neuroethics, Institute of Mental Health Research, University of Ottawa, Ottawa, Canada;

${ }^{4}$ Centre for Research Ethics \& Bioethics, University of Uppsala, Uppsala, Sweden

${ }^{5}$ Department of Psychology, Hangzhou Normal University, Hangzhou/China

E-mail address: Georg.Northoff@theroyal.ca (G. Northoff)

* Corresponding author: J. Shashi Kiran Reddy, NIAS, Indian Institute of Science (IISc) Campus. Email Address: jumpalreddy@live.com, jumpal shashi@yahoo.com 


\begin{abstract}
Antón-Bolaños et al. (2019) report a newly identified neural pathway mechanism, where the patterned spontaneous activity regulates the excitability of a neural network essential for the formation and maintenance of functional sensory maps in the brain. Findings from the study suggest that the patterned spontaneous activity prevalent during the embryonic development of the brain; at the early stages of innervation to the cortex, contributes to the formation of these sensory maps. Synesthesia is a neural phenomenon caused by the unusual links between sensory information, where synesthetic subjects demonstrate atypical functional and neural connectivity caused by the differences in cortical wiring during brain development. So, based on the findings from Antón-Bolaños et al. (2019), one can anticipate the role of spontaneous activity in promoting synesthetic condition. Thus, it will be interesting to study, if the intrinsic spontaneous activity influences the differential cortical wiring and the formation of sensory maps in synesthesia.
\end{abstract}

\title{
One Sentence Summary
}

The role of embryonic brain's spontaneous activity on synesthesia 


\section{Main Text}

A brief search for the brain regions recruited to write the present letter would reveal the active involvement of premotor cortex. Recent advancements in neuroscience identified such brain regions associated with various cognitive, affective and behavioural functions, yet, the mechanisms that trigger and govern the formation of such sensory maps in the brain remain unclear. To this end, Antón-Bolaños et al. (1) report a newly identified neural pathway mechanism, where the patterned spontaneous activity (SA) regulates the excitability of a neural network essential for the formation and maintenance of functional sensory maps in the brain. The patterned SA prevalent during the embryonic development of the brain; at the early stages of innervation to the cortex, contributes to the formation of these maps. Hitherto, it is thought that the formation of different sensory maps is primarily driven by molecular cues and subsequently by sensory experiences (2).

Findings from (1) will have direct clinical implications when employed to examine the potential pathways involved in a rare (nonpathological) neural condition known as synesthesia. This phenomenon is caused by the unusual links between sensory experiences triggered by the stimulation of multiple non-specialized cortices during a selective sensory encoding $(3,4)$. Synesthetes demonstrate atypical functional and neural connectivity which results from the differences in cortical wiring during the brain development. Although some potential gene expressions are also recently identified (4), as observed in (1), the role of SA can still be anticipated. Thus, it will be interesting to study, if the intrinsic SA influences the differential cortical wiring and the formation of sensory maps in synesthesia. 
In addition, considering the potential role of SA, future follow-up studies should examine the role of different parameters concerned with the intrinsic activity, such as the spatiotemporal repertoire, integration and speed. As suggested recently $(5,6)$, these parameters could establish the multisensory integration of different sensory information, which, even more importantly, are central for establishing mental features like consciousness and self.

\section{References}

1. N. Antón-Bolaños et al., Science (2019). 10.1126/science.aav7617.

2. P. Gaspar, N. Renier. Curr. Opin. Neurobiol. 53, 43-49 (2018).

3. R. E. Cytowic. Cambridge, Massachusetts: MIT Press (2002).

4. A. K. Tilot et al., PNAS 115 (12), 3168-3173 (2018).

5. G. Northoff et al., Phys Life Rev. (2019). https://doi.org/10.1016/j.plrev.2019.05.002

6. G. Northoff, Z. Huang. Neuroscience and Biobehavioral Reviews 80, 630-645 (2017). 